\title{
Analysis of secondary metabolites, antibacterial, brine shrimp lethality \& larvicidal potentiality of Microcos paniculata fruits
}

\author{
Md. Abdullah Aziz ${ }^{1 *}$, Ahmed Shafin Alam², Asma Akter Ema ${ }^{3}$, Monira Akter ${ }^{3}$, \\ Mohammed Motaher Hossain Chowdhury². \\ ${ }^{1}$ Lecturer, Department of Pharmacy, Stamford University Bangladesh. \\ ${ }^{2}$ Department of Pharmacy, Jahangirnagar University, Savar, Dhaka, Bangladesh. \\ ${ }^{3}$ Department of Pharmacy, Atish Dipankar University of Science \& Technology, Dhaka, Bangladesh.
}

\begin{abstract}
Microcos paniculata traditionally known as 'Kathgua or Fattashi' in Bangladesh is widely used for the treatment of various ailments including colds, diarrhea, hepatitis, heat stroke, dyspepsia, wound healing, fever and as an insecticide. Hence, the present study was designed to investigate the antibacterial, toxicity \& $4^{\text {th }}$ instar Culex quinquefasciatus mosquito larvicidal activities of fruit extracts of M. paniculata using a range of organic \& inorganic solvents (methanol, chloroform and water). Antibacterial, toxicity \& larvicidal effects were studied by means of agar disc diffusion, brine shrimp lethality bioassay $(B S L B) \&$ standard WHO protocol with slight modification. The susceptibility of the microorganisms to the plant extracts was compared with standard antibiotic flucloxacilin. Fruit methanol extract (FME) revealed a broad spectrum of antibacterial activity in contrast to other extracts (Fruit chloroform extract-FCE \& Fruit water extract-FWE), particularly significant against gram negative bacteria, Proteus mirabilis being the most (zone of inhibition $28 \mathrm{~mm}$ ). Moreover, the FME was found to be the most toxic to brine shrimp nauplii, with $L C_{50}$ of $52.7 \mu \mathrm{g} / \mathrm{ml}$, indicating the possibility of finding potential toxic compounds in this plant. $4^{\text {th }}$ instar Culex quinquefasciatus larval mortality was observed and recorded after $24 \mathrm{~h}$ exposure period. Both FME \& FCE exhibited considerable larvicidal effects with $L C_{50}$ of $342.1 \mu \mathrm{g} / \mathrm{ml} \& 441.7 \mu \mathrm{g} / \mathrm{ml}$ respectively. Altogether, these results propose that the organic fractions, FME \& FCE could be used as a potential sources of antibacterial agents along with their toxic \& larvicidal potentiality.
\end{abstract}

Keywords: Secondary metabolites, Antibacterial, Brine shrimp lethality, Larvicidal, Microcos paniculata.

\section{Introduction}

Application of medicinal plants for treatment of human ailments is a well-known phenomenon embedded in cultures of different communities of the developing world. Currently, there is resurgence in the use of herbal medicines globally; hence, implying increased efforts in evaluating these medicinal plants to establish their safety and efficacy. In developing countries, dependence on traditional medicines for primary healthcare needs has obvious reasons including, connections to cultural preference, but also largely due to limited modern health care facilities for at least up to $65 \%$ of the world population [1]. Infectious diseases still represent an important cause of morbidity and mortality among humans, especially in developing countries. Even though pharmaceutical companies have produced a number of new antibacterial drugs in the last few years, resistance to these drugs by microbes have increased and has now become a global concern. This situation forced scientists to search for new antimicrobial substances. Hence much attention has been paid to extracts and biologically active compounds isolated from plant species used in herbal medicine [2]. In order to study the toxicity of medicinal plants, the brine shrimp assay is considered a useful tool for preliminary assessment of toxicity and it has been used for the detection of fungal toxins, plant extract toxicity, heavy metals, pesticides and cytotoxicity testing of dental materials [3]. Mosquito-transmitted disease continues to be a major source of illness and death. Mosquitoes are also becoming increasingly resistant to traditional chemical pesticides and there is growing concern about the potential health and environmental risks surrounding these products. Environmental protection agencies have banned or placed severe restrictions on the use of many pesticides which were formerly used in mosquito control programmes [4]. Filariasis is an endemic, disabling, disfiguring disease and the filarial worm, Wuchereria bancrofti responsible for human filariasis is carried by Culex quinquefasciatus Say (Diptera: Culicidae), a pantropical pest and urban vector of Wuchereria bancrofti [5] and is probably the most abundant house mosquito in towns and cities of the tropical countries [6].

Microcos paniculata L. (English Name: Microcos; Family: Tiliaceae; Synonym: Microcos nervosa (Lour.) S. Y. Hu, Grewia nervosa (Lour.) Panigrahi) locally known as 'Kathgua or Fattashi' in Bangladesh. It is also known as Bu zha ye, Po bu ye (Transcribed Chinese);Kaphla (Transcribed Thai). It is a herbaceous plant which looks like a shrub or small tree that widely distributed and naturally grown throughout Bangladesh. It is also native and distributed more or less throughout India, Andaman and Nicobar (Andaman Islands), Sri Lanka, China, Cambodia, Myanmar, Thailand, Vietnam, Indonesia and Malaysia.M. paniculata L. is added in Chinese 
herb tea. It's claimed to have medicinal values as well. The taste of it is mildly sour. Traditional beliefs claim it services the digestive system to work better and it is additionally employed for other health conditions inclusive of colds ,diarrhea, hepatitis, heat stroke and dyspepsia. This plant traditionally used in wound healing, fever and as an insecticide in Bangladesh. Literature study reveals that the stem bark of M. paniculata contained a new alkaloid, N-Methyl-6 beta-(deca-1',3',5'-trienyl)-3 beta-methoxy-2 beta-methylpiperidine, which showed good insecticidal activity against Aedes aegypti second instar larvae. Another study claims that two new piperidine alkaloids, microcosamines A (1) and B (2), were isolated from the leaves of M. paniculata. Both new compounds showed significant larvicidal activity against Culex quinquefasciatus. On the other hand, the freeradical-scavenging assay of various solvent extracts of stem of Microcos paniculata yielded five compounds: a new triterpene named methyl 3beta-O-p-hydroxy-Ecinnamoyloxy- 2alpha,23-dihydroxyolean-12-en-28-oate (1), epicatechin (2), 3-trans-feruloyl maslinic acid (3), maslinic acid (4) and sucrose (5). Among them, compound 2 displayed significant free-radical-scavenging activity which is similar to that of standard antioxidant ascorbic acid and therefore may be a promising natural antioxidant. Beides, Rahman et al., 2011, investigated ethanolic extract of leaves of $M$. paniculata for cytotoxic activity and Aziz et al., 2013, studied secondary metabolites, antimicrobial, brine shrimp lethality $\& 4^{\text {th }}$ instar $C$. quinquefasciatus mosquito larvicidal screening of organic (methanol, chloroform) \& inorganic (water) root extracts of M. paniculata [7]. From the existing information it is evident that the plant may hold some important biological activities. However, considerably less is known about the chemical and biological works on the fruit of the plant.

Taking this in view and as a part of our ongoing research, the present study aimed to evaluate the antibacterial, toxicity and larvicidal activities against the $4^{\text {th }}$ instar larvae of $C$. quinquefasciatus filarial vector employing the FME, FCE \& FWE of M. paniculata.

\subsection{Collection and Identification of Plant Materials}

\section{Materials And Methods}

Fresh fruits of M. paniculata were collected from the Jahangirnagar University campus, Savar, Dhaka, Bangladesh in January, 2014 that were identified at the Bangladesh National Herbarium having the accession number 35348. A dried specimen was deposited in the herbarium.

\subsection{Preparation of the Plant Extracts}

Dried plant material $(180 \mathrm{~g})$ was used for extraction procedure. The extracts were all made with analytical grade solvents (Merck). The fruits were washed thoroughly 2-3 times with running water and once with sterile distilled water to remove the dust particles and then dried under shade for a period of 7 days. The dried plant materials were then ground into fine powders using a laboratory grinding mill. Plant samples were extracted with $900 \mathrm{ml}$ of methanol, chloroform and water separately using a soxhlet apparatus following hot extraction procedure. Then the extracts were filtered using Whatman No.1 filter papers. The filtrates were then dried in hot air oven at $40^{\circ} \mathrm{C}$. Then the extracts were stored under refrigeration at $4^{0} \mathrm{C}$ for further studies.

\subsection{Preliminary Secondary Metabolites Screening}

For preliminary secondary metabolites screening all the extractives were subjected to various tests (Table1) for determination of chemical nature of the extractives [8].

\subsubsection{Test Microorganisms and Preparation of Stock Culture}

The activity of plant extracts was tested on thirteen different organisms: four gram positive bacteria (Bacillus subtilis, Bacillus cereus, Bacillus megaterium, Staphylococcus aureus) and nine gram negative bacteria (Salmonella typhi, Vibrio cholerae, Proteus mirabilis, Escherichia coli, Serratia spp., Erwinia spp., Pseudomonas spp., Salmonella spp., Shigella boydii) were kindly provided by the Department of Microbiology, Jahangirnagar University, Savar, Dhaka, Bangladesh and reconfirmed by gram staining and sub culturing in appropriate selective media.

\subsubsection{Preparation of Standard Culture Inoculum of Test Microorganisms}

Three or four isolated colonies were inoculated in the $2 \mathrm{ml}$ nutrient broth and incubated till the growth in the broth was equivalent with Mac-Farland standard $(0.5 \%)$ as recommended by WHO.

\subsubsection{Antibacterial Assay}

Agar disc diffusion is a in vitro method for preliminary screening of test microorganisms [9]. The required amount of Petri plates are prepared and autoclaved at $121^{\circ} \mathrm{C}$ for 15 minutes. And they were allowed to cool under Laminarair flow. Aseptically transfer about $20 \mathrm{ml}$ of media into each sterile Petri dishes and allowed to solidify. $1 \mathrm{ml}$ inoculum suspension was spread uniformly over the agar medium using sterile glass rod to get 
uniform distribution of bacteria. The readily prepared sterile discs were loaded. The paper diffuse discs were placed on the medium suitably apart and the plate were incubated at $5^{\circ} \mathrm{C}$ for 1 hour to permit good diffusion and then transferred to an incubator at $37^{\circ} \mathrm{C}$ for 24 hours. The antibacterial activity was recorded by measuring the width of the clear inhibition zone around the disc using zone reader (mm).

\subsection{Brine Shrimp Lethality Bioassay}

The experiment was carried out using the method described by Meyer et al., 1982 [10]. In brief, Artemia salina Leach (brine shrimp eggs) was allowed to hatch and matured as nauplii (Larvae) in seawater for $48 \mathrm{hrs}$ at $25^{\circ} \mathrm{C}$. Serially diluted test solutions were added to the seawater, containing 10 nauplii. After incubation for $24 \mathrm{~h}$ at $25^{\circ} \mathrm{C}$, the number of survivors was counted. Vincristine sulfate was used as positive control.

\subsection{Larvicidal Bioassay}

The Larvicidal assay was carried as per standard WHO protocol with slight modification [11]. Various extracts of $M$. paniculata were investigated for their Larvicidal activity against $4^{\text {th }}$ instar larvae of $C$. quinquefasciatus. In the present study, for treatment of larvae with the fruit extracts of M. paniculata, $100 \mathrm{ml}$ of tap water was kept in a series of glass beakers (of $200 \mathrm{ml}$ capacity). Required quantity of stock solution was added into each beaker (containing $100 \mathrm{ml}$ of tap water) to obtain a particular concentration of the extracts. Control medium was also maintained with $100 \mathrm{ml}$ of tap water Separate series of exposure medium with desired concentrations of extract were kept for $C$. quinquefasciatus. The larval mortality of fourth instar larvae of $C$. quinquefasciatus were observed separately in control, 50, 100, 150, 200, 250, 300, 350, 400, 450 and 500 ppm (parts per million) concentrations. Ten numbers of $4^{\text {th }}$ instar larvae of $C$. quinquefasciatus were separately introduced into control and different concentrations of leaf extract. The number of alive and dead larvae at the end of $24 \mathrm{~h}$, response $\%$, corrected response $\%$, linear response $\%$, linear probit, $\mathrm{LC}_{50}, \mathrm{LC}_{90}$, lower limit \& upper limit and calculated chi-square values were recorded.

\section{Statistical Analysis}

The $\mathrm{LC}_{50}(50 \%$ lethal concentration, $\mu \mathrm{g} / \mathrm{ml})$ of different plant extracts were determined from duplicate experiments. $\mathrm{LC}_{90}(90 \%$ lethal concentration, $\mu \mathrm{g} / \mathrm{ml})$, and other statistics at 95 percent fiducial limits of upper confidence limit and lower confidence limit, and chi-square values were calculated by using $\operatorname{LdP}_{\text {Line }}^{\mathrm{R}}$ software [12].

\section{Results}

The crude extracts when tested with various chemical reagents demonstrated the presence of alkaloids, flavonoids, saponins and steroids shown in Table-1.

The antibacterial activity of specific concentrations of different extracts of $M$. paniculata is given in Table-2.The crude extracts of plant showed effective antibacterial activities. All the plant extracts showed dose dependent inhibition against maximum bacteria. Among the extracts, FME showed highest efficacy against most of the bacteria and satisfactory inhibition against the gram negative bacteria selected, $P$. mirabilis being the most. The standard, flucloxacilin, revealed noteworthy zone of inhibition for the maximum test organisms. Gram positive bacteria were inhibited more than gram negative bacteria by flucloxacilin. In addition, $V$. cholerae being the most inhibited organism, though it is a gram negative bacteria. In case of BSLB, FME was found to be the most toxic than other extracts to Brine Shrimp nauplii, with $\mathrm{LC}_{50}$ of $52.7 \mu \mathrm{g} / \mathrm{ml}$, whereas the anticancer drug VS (Vincristine sulphate) showed $\mathrm{LC}_{50}$ value $1.7 \mu \mathrm{g} / \mathrm{ml}$. The order at which toxicity potential of the test samples decreased was as follows: Vincristine sulphate $>$ FME $>$ FCE $>$ FWE.

The results of various extracts of $M$. paniculata were screened for their larvicidal activity against the $4^{\text {th }}$ instar larvae of $C$. quinquefasciatus at the end of $24 \mathrm{~h}$ are represented in (Table 7-9). Among the three extracts screened, FME showed promising larvicidal effects. Remaining extracts also showed considerable larvicidal activities. Based on the Probit analysis, the $24 \mathrm{~h} \mathrm{LC}_{50}$ value of FME for C. quinquefasciatus was found to $342.1 \mathrm{ppm}$ (Table-7).

Table-1: Secondary metabolites screening of various extracts of fruits of $M$. paniculata.

\begin{tabular}{|c|c|c|c|}
\hline \multirow{2}{*}{ Compounds } & \multicolumn{3}{|c|}{ Observation of various extracts } \\
\cline { 2 - 4 } & Methanol & Chloroform & Water \\
\hline Alkaloid & + & - & + \\
\hline Saponin & + & - & - \\
\hline Tannin & - & - & - \\
\hline Flavonoid & - & + & + \\
\hline Steroid & + & + & - \\
\hline Glucoside & - & - & \\
\hline
\end{tabular}




\begin{tabular}{|c|c|c|c|}
\hline Glycoside & - & - & - \\
\hline
\end{tabular}

$(+)=$ Present; $(-)=$ absent

Table-2: Antibacterial activity of $M$. paniculata at 2, 4 and $6 \mathrm{mg} /$ disc including standard flucloxacilin at

\begin{tabular}{|c|c|c|c|c|c|c|c|c|c|c|}
\hline & & & & & $g /$ & & & & & \\
\hline \multirow[t]{2}{*}{ Organisms } & \multicolumn{3}{|c|}{ FME } & \multicolumn{3}{|c|}{ FCE } & \multicolumn{3}{|c|}{ FWE } & \multirow{2}{*}{$\begin{array}{r}\text { Flucloxacilin } \\
(10 \mu \mathrm{g} / \mathrm{disc})\end{array}$} \\
\hline & $2 \mathrm{mg}$ & $4 \mathrm{mg}$ & mg & $2 \mathrm{mg}$ & $\operatorname{lmg} 6$ & & $2 \mathrm{mg}$ & $4 \mathrm{mg}$ & $6 \mathrm{mg}$ & \\
\hline \multicolumn{11}{|c|}{ Diameter of Zone of Inhibition (mm) } \\
\hline B. subtilis & 7.5 & 16 & 20 & 7 & 13.5 & 19 & 7 & 10 & 14 & 25 \\
\hline B. cereus & 9 & 11 & 13 & 6.5 & 12 & 18 & - & 10 & 16 & 23 \\
\hline B. megaterium & - & 6.5 & 10 & 6 & 12 & 17 & - & 7 & 13 & 13.5 \\
\hline S. typhi & 12 & 14 & 19 & 9 & 13 & 18 & 10 & 19 & 20 & - \\
\hline V. cholerae & 14 & 14 & 17 & - & 7 & 14 & 12 & 17 & 19 & 27 \\
\hline P. mirabilis & 19 & 25 & 28 & 10 & 18 & 25 & 7 & 18 & 18 & 10 \\
\hline E. coli & 11 & 13 & 21 & 6.5 & 11 & 17 & 6 & 9 & 12 & 13 \\
\hline S. aureus & 7 & 14 & 19 & - & 7 & 13 & 6 & 7 & 8 & 20 \\
\hline Serratia spp. & 9 & 9 & 15 & 7 & 7 & 15 & 6 & 7.5 & 9 & - \\
\hline Erwinia spp. & 20 & 22 & 24 & - & 8 & 12 & 6 & 6.5 & 7 & 20 \\
\hline Pseudomonas spp. & 15 & 19 & 20 & 7.5 & 11 & 14 & 7 & 14 & 22 & - \\
\hline Salmonella spp. & 11 & 13 & 16 & - & 8 & 16 & 6 & 8.5 & 12 & 17.5 \\
\hline Shigella boydii & 9 & 15 & 18 & - & 8 & 13 & 11 & 16 & 17 & 14 \\
\hline
\end{tabular}

Table-3: Probit analysis, $\mathrm{LC}_{50}, \mathrm{LC}_{90} \&$ calculated chi square $\left(\chi^{2}\right)$ values for FME.

\begin{tabular}{|c|c|c|c|c|c|c|c|c|}
\hline$\underset{)}{\text { Conc. }(\mu \mathrm{g} / \mathrm{ml}}$ & Treated & Response \% & $\begin{array}{l}\text { Corrected } \\
\text { response \% }\end{array}$ & $\begin{array}{c}\text { Linear } \\
\text { response } \%\end{array}$ & Linear probit & $\mathrm{LC}_{\mathrm{s} 0}(\mu \mathrm{g} / \mathrm{ml})$ & $\underset{\mathrm{LC}_{90}(\mu \mathrm{g} / \mathrm{ml}}{ }$ & $x^{2}$ \\
\hline 1 & 20 & 20 & 15.8 & 6.4 & 3.5 & \multirow{8}{*}{$\begin{array}{c}\mathrm{LC}_{50}= \\
52.7 \\
\text { Lower } \\
\text { limit=30.0 } \\
\text { Upper } \\
\text { limit=100.2 }\end{array}$} & \multirow{8}{*}{$\begin{array}{c}C_{p 0}=1485 . \\
171 \\
\text { Lower } \\
\text { limit=528.8 } \\
6 \\
\text { Upper } \\
\text { limit=1017 } \\
1.1127\end{array}$} & \multirow[t]{8}{*}{8.0} \\
\hline 5 & 20 & 20 & 15.8 & 18.3 & 4.1 & & & \\
\hline 10 & 20 & 20 & 15.8 & 26.1 & 4.4 & & & \\
\hline 20 & 20 & 40 & 36.8 & 35.5 & 4.6 & & & \\
\hline 50 & 20 & 50 & 47.4 & 49.2 & 5.0 & & & \\
\hline 100 & 20 & 50 & 47.4 & 59.7 & 5.2 & & & \\
\hline 200 & 20 & 70 & 68.4 & 69.6 & 5.5 & & & \\
\hline 500 & 20 & 95 & 94.7 & 80.6 & 5.9 & & & \\
\hline
\end{tabular}

Where Conc. $=$ Concentration.

Table-4: Probit analysis, $\mathrm{LC}_{50}, \mathrm{LC}_{90} \& \chi^{2}$ values for FCE.

\begin{tabular}{|c|c|c|c|c|c|c|c|c|}
\hline $\begin{array}{c}\text { Conc. }(\mu \mathrm{g} / \\
\mathrm{ml})\end{array}$ & Treated & $\begin{array}{c}\text { Response } \\
\%\end{array}$ & $\begin{array}{l}\text { Corrected } \\
\text { response } \%\end{array}$ & $\begin{array}{c}\text { Linear } \\
\text { response } \\
\%\end{array}$ & $\begin{array}{l}\text { Linear } \\
\text { probit }\end{array}$ & $\begin{array}{c}\mathrm{LC}_{50}(\mu \mathrm{g} / \\
\mathrm{ml})\end{array}$ & $\begin{array}{c}\mathrm{LC}_{90}(\mu \mathrm{g} / \\
\mathrm{ml})\end{array}$ & $\chi^{2}$ \\
\hline 1 & 20 & 10 & 5.3 & 4.7 & 3.3 & \multirow{8}{*}{$\begin{array}{c}\mathrm{LC}_{50}= \\
150.9 \\
\text { Lower } \\
\text { limit }=76.7 \\
\text { Upper } \\
\text { limit }=444 . \\
5\end{array}$} & \multirow{8}{*}{$\begin{array}{c}\mathrm{LC}_{90}=69 \\
77.4 \\
\text { Lower } \\
\text { limit }=15 \\
65.4 \\
\text { Upper } \\
\text { limit }=17 \\
4259.8\end{array}$} & \multirow[t]{8}{*}{10.7} \\
\hline 5 & 20 & 20 & 15.8 & 12.7 & 3.9 & & & \\
\hline 10 & 20 & 30 & 26.3 & 18.2 & 4.1 & & & \\
\hline 20 & 20 & 30 & 26.3 & 25.0 & 4.3 & & & \\
\hline 50 & 20 & 30 & 26.3 & 35.6 & 4.6 & & & \\
\hline 100 & 20 & 30 & 26.3 & 44.5 & 4.9 & & & \\
\hline 200 & 20 & 45 & 42.1 & 53.5 & 5.1 & & & \\
\hline 500 & 20 & 90 & 89.4 & 65.6 & 5.4 & & & \\
\hline
\end{tabular}

Table-5: Probit analysis, $\mathrm{LC}_{50}, \mathrm{LC}_{90} \& \chi^{2}$ values for $\mathrm{FWE}$ 


\begin{tabular}{|c|c|c|c|c|c|c|c|c|}
\hline $\begin{array}{l}\text { Conc. }(\mu \mathrm{g} / \\
\mathrm{ml})\end{array}$ & Treated & $\begin{array}{c}\text { Response } \\
\%\end{array}$ & $\begin{array}{l}\text { Corrected } \\
\text { response \% }\end{array}$ & $\begin{array}{c}\text { Linear } \\
\text { response } \\
\%\end{array}$ & $\begin{array}{l}\text { Linear } \\
\text { probit }\end{array}$ & $\begin{array}{c}\mathrm{LC}_{50}(\mu \mathrm{g} / \\
\mathrm{ml})\end{array}$ & $\begin{array}{c}\mathrm{LC}_{90}(\mu \mathrm{g} / \\
\mathrm{ml})\end{array}$ & $\chi^{2}$ \\
\hline 1 & 20 & 20 & 15.8 & 18.3 & 4.1 & \multirow[t]{8}{*}{$\begin{array}{c}\mathrm{LC}_{50}= \\
24225.6\end{array}$} & \multirow{8}{*}{$\begin{array}{c}\mathrm{LC}_{90}=40 \\
0535920 \\
56.053\end{array}$} & \multirow[t]{8}{*}{0.7} \\
\hline 5 & 20 & 30 & 26.3 & 22.4 & 4.2 & & & \\
\hline 10 & 20 & 30 & 26.3 & 24.3 & 4.3 & & & \\
\hline 20 & 20 & 30 & 26.3 & 26.3 & 4.4 & & & \\
\hline 50 & 20 & 30 & 26.3 & 29.0 & 4.4 & & & \\
\hline 100 & 20 & 30 & 26.3 & 31.2 & 4.5 & & & \\
\hline 200 & 20 & 40 & 36.8 & 33.4 & 4.6 & & & \\
\hline 500 & 20 & 40 & 36.8 & 36.4 & 4.7 & & & \\
\hline
\end{tabular}

For the above data (Table -5), lower limit \& upper limit of $\mathrm{LC}_{50} \& \mathrm{LC}_{90}$ couldn't be calculated by LdP Line ${ }^{\mathrm{R}}$ software as ' $\mathrm{g}$ ' value was greater than 0.4. ' $\mathrm{g}$ ' is a factor used in fiducial limit calculations [12]. "With almost all good sets of data, 'g' will be substantially smaller than 0.1 , and seldom greater than 0.4 " [13].

Table-6: Probit analysis, $\mathrm{LC}_{50}, \mathrm{LC}_{90} \& \chi^{2}$ values for VS.

\begin{tabular}{|c|c|c|c|c|c|c|c|c|}
\hline $\begin{array}{l}\text { Conc. }(\mu \mathrm{g} / \\
\mathrm{ml})\end{array}$ & Treated & $\begin{array}{c}\text { Response } \\
\%\end{array}$ & $\begin{array}{l}\text { Corrected } \\
\text { response \% }\end{array}$ & $\begin{array}{c}\text { Linear } \\
\text { response } \\
\%\end{array}$ & $\begin{array}{l}\text { Linear } \\
\text { probit }\end{array}$ & $\begin{array}{c}\mathrm{LC}_{50}(\mu \mathrm{g} / \\
\mathrm{ml})\end{array}$ & $\begin{array}{c}\mathrm{LC}_{90}(\mu \mathrm{g} / \\
\mathrm{ml})\end{array}$ & $\chi^{2}$ \\
\hline 0.06 & 20 & 10 & 5.3 & 3.5 & 3.2 & \multirow{8}{*}{$\begin{array}{c}\mathrm{LC}_{50}= \\
1.7 \\
\text { Lower } \\
\text { limit }=1.1 \\
\text { Upper } \\
\text { limit }=2.9\end{array}$} & \multirow{8}{*}{$\begin{array}{c}\text { LC }_{90}=18 . \\
7 \\
\text { Lower } \\
\text { limit }=9.4 \\
\text { Upper } \\
\text { limit }=54 . \\
7\end{array}$} & \multirow[t]{8}{*}{3.3} \\
\hline 0.125 & 20 & 15 & 10.5 & 7.8 & 3.6 & & & \\
\hline 0.25 & 20 & 15 & 10.5 & 14.8 & 4.0 & & & \\
\hline 0.5 & 20 & 25 & 21.1 & 25.2 & 4.3 & & & \\
\hline 1 & 20 & 45 & 42.1 & 38.3 & 4.7 & & & \\
\hline 5 & 20 & 65 & 63.2 & 71.6 & 5.6 & & & \\
\hline 12.5 & 20 & 95 & 94.7 & 85.6 & 6.1 & & & \\
\hline 25 & 20 & 95 & 94.7 & 92.5 & 6.4 & & & \\
\hline
\end{tabular}

Table-7: Response $\%$, corrected response $\%$, linear response $\%$ and linear probit of $4^{\text {th }}$ instar larvae of $C$. quinquefasciatus exposed for 24 hours to different concentrations for FME of M. paniculata. 


\begin{tabular}{|c|c|c|c|c|c|c|c|c|}
\hline $\begin{array}{l}\text { Conc. } \\
\text { (PPM) }\end{array}$ & Treated & $\begin{array}{c}\text { Response } \\
\%\end{array}$ & $\begin{array}{c}\text { Corrected } \\
\text { response } \\
\%\end{array}$ & $\begin{array}{c}\text { Linear } \\
\text { response } \\
\%\end{array}$ & $\begin{array}{l}\text { Linear } \\
\text { probit }\end{array}$ & $\begin{array}{c}\mathrm{LC}_{50}(\mu \mathrm{g} / \mathrm{m} \\
\mathrm{l})\end{array}$ & $\begin{array}{c}\mathrm{LC}_{90}(\mu \mathrm{H} \\
\mathrm{g} / \mathrm{ml})\end{array}$ & $\chi^{2}$ \\
\hline 50 & 20 & 10 & 5.3 & 0.4 & 2.4 & \multirow{10}{*}{$\begin{array}{c}\mathrm{LC}_{50}= \\
342.1 \\
\text { Lower } \\
\text { limit }=262.8 \\
\text { Upper } \\
\text { limit }=521.8\end{array}$} & \multirow{10}{*}{$\begin{array}{c}\text { LC }_{90}=8 \\
67.3 \\
\text { Lower } \\
\text { limit }=7 \\
64.9 \\
\text { Upper } \\
\text { limit }=3 \\
175.3\end{array}$} & \multirow[t]{10}{*}{19.7} \\
\hline 100 & 20 & 10 & 5.3 & 4.5 & 3.3 & & & \\
\hline 150 & 20 & 15 & 10.5 & 12.8 & 3.9 & & & \\
\hline 200 & 20 & 15 & 10.5 & 23.0 & 4.3 & & & \\
\hline 250 & 20 & 25 & 21.1 & 33.3 & 4.6 & & & \\
\hline 300 & 20 & 35 & 31.6 & 42.8 & 4.8 & & & \\
\hline 350 & 20 & 70 & 68.4 & 51.3 & 5.0 & & & \\
\hline 400 & 20 & 65 & 63.2 & 58.5 & 5.2 & & & \\
\hline 450 & 20 & 65 & 63.2 & 64.7 & 5.4 & & & \\
\hline 500 & 20 & 80 & 78.9 & 70.0 & 5.5 & & & \\
\hline
\end{tabular}

Table-8: Response $\%$, corrected response $\%$, linear response $\%$ and linear probit of $4^{\text {th }}$ instar larvae of $C$. quinquefasciatus exposed for 24 hours to different concentrations for FCE of M. paniculata.

\begin{tabular}{|c|c|c|c|c|c|c|c|c|}
\hline $\begin{array}{l}\text { Conc. } \\
\text { (PPM) }\end{array}$ & Treated & $\begin{array}{c}\text { Response } \\
\%\end{array}$ & $\begin{array}{c}\text { Corrected } \\
\text { response } \\
\%\end{array}$ & $\begin{array}{c}\text { Linear } \\
\text { response } \\
\%\end{array}$ & $\begin{array}{l}\text { Linear } \\
\text { probit }\end{array}$ & $\begin{array}{c}\mathrm{LC}_{50}(\mu \mathrm{g} / \mathrm{m} \\
\mathrm{l})\end{array}$ & $\begin{array}{c}\mathrm{LC}_{90}(\mu \\
\mathrm{g} / \mathrm{ml})\end{array}$ & $\chi^{2}$ \\
\hline 50 & 20 & 10 & 5.3 & 0.5 & 2.4 & \multirow{10}{*}{$\begin{array}{c}\text { LC }_{50}= \\
441.7 \\
\text { Lower } \\
\text { limit }=366.5 \\
\text { Upper } \\
\text { limit }=604.1\end{array}$} & \multirow{10}{*}{$\begin{array}{c}\mathrm{LC}_{90}=1 \\
315.3 \\
\text { Lower } \\
\text { limit }=8 \\
56.0 \\
\text { Upper } \\
\text { limit }=3 \\
301.2\end{array}$} & \multirow[t]{10}{*}{14.7} \\
\hline 100 & 20 & 10 & 5.3 & 4.1 & 3.3 & & & \\
\hline 150 & 20 & 10 & 5.3 & 10.2 & 3.7 & & & \\
\hline 200 & 20 & 20 & 15.8 & 17.6 & 4.1 & & & \\
\hline 250 & 20 & 20 & 15.8 & 25.2 & 4.3 & & & \\
\hline 300 & 20 & 25 & 21.1 & 32.5 & 4.5 & & & \\
\hline 350 & 20 & 40 & 36.8 & 39.2 & 4.7 & & & \\
\hline 400 & 20 & 45 & 42.0 & 45.4 & 4.9 & & & \\
\hline 450 & 20 & 60 & 57.9 & 50.9 & 5.0 & & & \\
\hline 500 & 20 & 75 & 73.7 & 50.8 & 5.1 & & & \\
\hline
\end{tabular}

Table-9: Response $\%$, corrected response $\%$, linear response $\%$ and linear probit of $4^{\text {th }}$ instar larvae of $C$. quinquefasciatus exposed for 24 hours to different concentrations for FWE of M. paniculata. 


\begin{tabular}{|c|c|c|c|c|c|c|c|c|}
\hline $\begin{array}{l}\text { Conc. } \\
\text { (PPM) }\end{array}$ & Treated & $\begin{array}{c}\text { Response } \\
\%\end{array}$ & $\begin{array}{c}\text { Corrected } \\
\text { response } \\
\%\end{array}$ & $\begin{array}{c}\text { Linear } \\
\text { response } \\
\%\end{array}$ & $\begin{array}{l}\text { Linear } \\
\text { probit }\end{array}$ & $\begin{array}{c}\mathrm{LC}_{50}(\mu \mathrm{g} / \mathrm{m} \\
\mathrm{l})\end{array}$ & $\begin{array}{c}\mathrm{LC}_{90}(\mu \\
\mathrm{g} / \mathrm{ml})\end{array}$ & $\chi^{2}$ \\
\hline 50 & 20 & 10 & 5.3 & 2.4 & 3.0 & \multirow{10}{*}{$\begin{array}{c}\mathrm{LC}_{50}= \\
1185.6 \\
\text { Lower } \\
\text { limit }=642.7 \\
\text { Upper } \\
\text { limit }=1197 \\
3.6\end{array}$} & \multirow{10}{*}{$\begin{array}{c}\mathrm{LC}_{90}=9 \\
234.0 \\
\text { Lower } \\
\text { limit }=2 \\
389.1 \\
\text { Upper } \\
\text { limit }=2 \\
138936 . \\
9\end{array}$} & \multirow[t]{10}{*}{1.7} \\
\hline 100 & 20 & 10 & 5.3 & 6.1 & 3.5 & & & \\
\hline 150 & 20 & 13 & 8.8 & 9.8 & 3.7 & & & \\
\hline 200 & 20 & 15 & 10.5 & 13.3 & 3.9 & & & \\
\hline 250 & 20 & 20 & 15.8 & 16.6 & 4.0 & & & \\
\hline 300 & 20 & 20 & 15.8 & 19.5 & 4.1 & & & \\
\hline 350 & 20 & 25 & 21.1 & 22.3 & 4.2 & & & \\
\hline 400 & 20 & 30 & 26.3 & 24.9 & 4.3 & & & \\
\hline 450 & 20 & 30 & 26.3 & 27.3 & 4.4 & & & \\
\hline 500 & 20 & 40 & 36.8 & 29.5 & 4.5 & & & \\
\hline
\end{tabular}

\section{Discussion}

Multiple antibiotic resistances in pathogenic bacteria has become a serious matter of concern, as reasons like natural evolutionary modes of obtaining both intrinsic and extrinsic resistance patterns [14].

Concomitant to search for new generation of drugs for increased MDR (Multi Drug Resistant) pathogenic bacteria, continual efforts for search of control agents from plants and related sources have been undertaken.

M. paniculata is one of the medicinal plants that are commonly used by the local practitioners for various human ailments, but no attempt has been made to study the antibacterial activity of its fruits.

Gram negative bacteria \& mycobacteria both possess thick outer membranes that are highly hydrophobic, providing these organisms with a permeability barrier especially towards hydrophilic compounds such as macrolide antibiotics such as erythromycin. The genus, Staphylococcus includes pathogenic organisms of which, $S$. aureus is frequently isolated from clinical samples. This species has overcome the control from most modern therapeutic agents; eventually its control has become the most hazardous. Further, beta-lactamase activity of $S$. aureus has caused resistance to most penicillin derivatives including the oxacillin and now is identified as MRSA, which has become the major the cause of nosocomial infections worldwide, including India [15].

The present study showed that FME of $M$. paniculata inhibited the gram negative bacteria better than gram positive bacteria. Phytochemical constituents such as alkaloids, flavonoids, tannins, phenols, saponins, and several other aromatic compounds are secondary metabolites of plants that serve a defense mechanism against prediction by many microorganisms, insects and other herbivores [16].

These bioactive compounds are known to act by different mechanism and exert antimicrobial action. Flavonoids are hydroxylated phenolic substance known to be synthesized by plants in response to microbial infection and it should not be surprising that they have been found in vitro to be effective antimicrobial substances against a wide array of microorganisms. Their activity is probably due to their ability to complex with extracellular and soluble proteins and to complex with bacterial cell walls [17]. Antimicrobial property of saponin is due to its ability to cause leakage of proteins and certain enzymes from the cell [18]. Steroids have been reported to have antibacterial properties, the correlation between membrane lipids and sensitivity for steroidal compound indicates the mechanism in which steroids specifically associate with membrane lipid and exerts its action by causing leakages from liposomes [19].

The higher antimicrobial activity of the methanolic extract as compared to aqueous \& chloroform extracts might be due to the lack of solubility of active constituents in aqueous solution. It is clear that the effectiveness of the extracts largely depends on the type of solvent used. The organic extracts provided more powerful antibacterial activities as compared to aqueous extract. This observation clearly indicates that the existence of non-polar residues in the extracts that are hydrophobic in nature, have higher antibacterial abilities against the gram negative bacteria.

Brine shrimp lethality bioassay is used in the bioassay for the bioactive compounds [10, 20]. In toxicity evaluation of plant extracts by brine shrimp lethality bioassay $\mathrm{LC}_{50}$ values lower than $1000 \mu \mathrm{g} / \mathrm{ml}$ are considered 
bioactive [10]. The Brine Shrimp Lethality Bioassay also indicates antifungal effects, pesticidal effects, teratogenic effects, toxicity to environment and many more [21].

Tables 3,4,5 show the lethality of different extracts of M. paniculata to the Brine Shrimp nauplii. The degree of lethality shown by the extractives was found to be directly proportional to the concentration of the extractives ranging from the lowest concentration $(1 \mu \mathrm{g} / \mathrm{ml})$ to the highest concentration $(500 \mu \mathrm{g} / \mathrm{ml})$. This concentration dependent increment in percent mortality of Brine Shrimp nauplii produced by the M. paniculata indicates the possibility of finding toxic compounds in these extractives. Preliminary secondary metabolites screening revealed the presence of alkaloids (except FCE) and steroids. So the observed toxicity action may be due to the presence of such compounds. Again, reports exist on the role of alkaloids and steroids in cytotoxic activity of plant extracts [22, 23, 24].

Moreover, this significant lethality of the plant extracts $\left(\mathrm{LC}_{50}\right.$ values less than $\left.151 \mu \mathrm{g} / \mathrm{ml}\right)$ apart from FWE $\left(\mathrm{LC}_{50}=24225.6 \mu \mathrm{g} / \mathrm{ml}\right)$ to brine shrimp is indicative of the presence of powerful toxic and probably insecticidal compounds which warrants further investigation.

Vector-borne diseases constitute the major cause of morbidity in most of the tropical and subtropical countries and have always been a challenge to the medical professionals struggling for the welfare of humanity. Mosquitoes are the most deadly vector for several of these disease causing organisms. In many parts of the world, plant-derived natural products have traditionally been used against mosquitoes [7].

However, resistance and environment damage caused by synthetic agents have prompted for the search for environmental friendly and effective larvicides from plant sources [25].

The findings of the existing analysis revealed that the FME part of $M$. paniculata contained alkaloid, steroid and FCE showed the presence of alkaloid, flavonoid and steroid that may be responsible for their (except $\mathrm{FWE}, \mathrm{LC}_{50}=1185.6 \mu \mathrm{g} / \mathrm{ml}$ ) potential larvicidal activities against $4^{\text {th }}$ instar larvae of $C$. quinquefasciatus.

\section{Conclusion}

From the above study, the integrated data suggested that the plant, $M$. paniculata has efficiency against bacteria both gram positive and gram negative and good toxicity \& larvicidal activities also. This study will guide researchers for the isolation of new compounds from M. paniculata that will provide good efficacy for the ailments of infections and diseases.

\section{Acknowledgments}

The authors would like to thank Professor Abdullah Faruque, Department of Pharmacy, Jahangirnagar University, Savar, Dhaka, Bangladesh \& Associate Professor Dr. Kabirul Bashar, Department of Zoology, Jahangirnagar University, Savar, Dhaka, Bangladesh for providing facilities and encouragement.

\section{References}

[1]. Daniel, S.F., Norman, R.F., 2001. The value of plants used in traditional medicine for drug discovery. Environ. Health Perspect. 109, 69-75.

[2]. Essawi, T., Srour, M., 2000. Screening of some Palestinian medicinal plants for antibacterial activity. J. Ethnopharmacol. 70, 343 349.

[3]. Krishnaraju, A.V., Rao, T.V.N., Sundararaju, D., Vanisree, M., Tsay, H.S., Subbaraju, G.V., 2005. Assessment of bioactivity of Indian medicinal plants using brine shrimp (Artemia salina) lethality assay. Int. J. Appl. Sci. Eng. 3, 125-134

[4]. Aarthi, N., Murugan, K., 2010. Larvicidal and smoke repellent activities of Spathodea campanulata P. Beauv. Against the malarial vector Anopheles Stephensi LIS (Diptera:Culicidae). J. Phytol. 2, 61-69.

[5]. Holder, P.W., Brown, G., Bullians, M., 1999. The mosquitoes of New Zealand and their animal disease significance. Surveillance. 26(4), 12-15.

[6]. Samuel, T., Arivoli, S., Martin J. J., 2011. Larvicidal efficacy of Vernonia cinerea (L.) (Asteraceae) leaf extracts against the filarial vector Culex quinquefasciatus Say (Diptera: Culicidae). J. Biopest. 4 (1), $37-42$.

[7]. Aziz, M.A., Shawn, M.M.A.K., Rahman, S., Islam, T., Mita, M., Faruque, A., Rana, M.S., 2013. Secondary metabolites, antimicrobial, brine shrimp lethality $\& 4^{\text {th }}$ instar Culex quinquefasciatus mosquito larvicidal screening of organic \& inorganic root extracts of Microcos paniculata. IOSR-JPBS. 8, 58-65.

[8]. Ghani, A., 2003. Medicinal Plants of Bangladesh. $2^{\text {nd }}$ Ed. The Asiatic Society of Bangladesh. Dhaka, Bangladesh. pp. 31, 39-40, 418, 500-504, 589-580.

[9]. Bayer, A.W., Kirby, W.M.M., Sherris, J.C., Turck, M., 1966. Antibiotic susceptibility testing by a standardized single disc method. Am. J. Clin. Pathol. 45,493-496.

[10]. Meyer, B.N., Ferrigni, N.R., Putnam, J.E., Jacobsen, L.B., Nichols, D.E., Mclaughlin, J.L., 1982. Brine shrimp: a convenient general bioassay for active plant constituents. Planta Med. 45, 31-34.

[11]. WHO. Report of the WHO informal consultation on the evaluation and testingof insecticides. Geneva: WHO, 1996.

[12]. Ehabsoft [http://www.ehabsoft.com/ldpline].

[13]. Finney, D.J., 1972. Probit Analysis. $3^{\text {rd }}$ Ed. Cambridge University Press, Cambridge, England . pp. 79.

[14]. Dubey, D., Sahu, M.C., Rath, S., Paty, B.P., Debata, N.K., Padhy, R.N., 2012. Antimicrobial activity of medicinal plants used by aborigines of Kalahandi, Orissa, India against multidrug resistant bacteria. Asian Pac. J. Trop. Biomed. 2, S846-S854.

[15]. Chitemerere, T.A., Mukanganyama, S., 2011. In vitro antibacterial activity of selected medicinal plants from Zimbabwe. African J. Plant Sci. Biotech. 5,1-7. 
[16]. Bonjar, G.H.S., Nik, A.K., Aghighi, S., 2004. Antibacterial and antifungal survey in plants used in indigenous herbal-medicine of south east regions of Iran. J. Biol. Sci. 4, 405-412.

[17]. Cowan, M.M., 1999. Plant products as antimicrobial agents. Clin. Microbiol. Rev. 12, 564-582.

[18]. Zablotowicz, R.M., Hoagland, R.E., Wagner, S.C., 1996. Effect of saponins on the growth and activity of rhizosphere bacteria. Adv. Exp. Med. Biol. 405, 83-95.

[19]. Madduluri, S., Rao, K., Sitaram, B., 2013. In vitro evaluation of antibacterial activity of five indigenous plants extract against five bacterial pathogens of human. Int. J. Pharm. Pharm. Sci. 5, 679-684.

[20]. Zhao, G.X., Hui, Y.H., Rupprecht, J.K., McLaughlin, J.L., Wood, K.V., 1992. Additional bioactive compounds and trilobacin, a novel highly cytotoxic acetogenin, from the bark of Asimina triloba. J. Natl. Prod. 55, 347-356.

[21]. Vanhaecke, P., Persoone, G., Claus, C., Sorgeloos, P., 1981. Proposal for a short-term toxicity test with Artemia nauplii. Eco. Toxicol. Environ. Safety. 5, 382-387.

[22]. Badami, S., Manohara, S.A., Kumar, E.P., 2003. Antitumor activity of total alkaloid fraction of Solanum pseudocapsicum leaves. Phytother. Res. 17, 1001-1004.

[23]. Dhar, M.L., Dhar, M.N., Dhawan, B.N., 1973. Screening of Indian medicinal plants for biological activity. Ind. J. Expt. Biol. 11, 43-45.

[24]. Vijayan, P., Rreethi, V., Prashanth, S.H., Raghu, H., 2004. Cytotoxicity activity of the total alkaloids isolated from different parts of Solanum pseudocapsicum. Biol. Pharm. Bull. 24, 528-530.

[25]. Gu, H.J., Cheng, S.S., Huang, C.G., Chen, W.J., Chang, S.T., 2009. Mosquito larvicidal activities of extractives from black heartwood-type Cryptomeria japonica. Parasitol. Res. 105, 1455-1458. 